\title{
Label Preference Schemes in GMPLS Controlled Networks
}

\author{
N. Andriolli, J. Buron, Student Member, IEEE, S. Ruepp, Student Member, IEEE, F. Cugini, \\ L. Valcarenghi, Member, IEEE, and P. Castoldi, Member, IEEE
}

\begin{abstract}
The GMPLS assumption that all available labels are equal is reasonable in electronic networks but not always true in WDM optical networks where labels correspond to physical wavelengths. In this paper we present two schemes for collecting the preference for specific labels during GMPLS signaling. For this purpose a new use of the Suggested Label object is proposed, and a novel object called Suggested Vector is introduced. The approach is validated through simulations showing significant wavelength converter usage reduction in a WDM optical network.
\end{abstract}

Index Terms- GMPLS signaling, label preference.

\section{INTRODUCTION}

$\mathbf{T}$ HE Generalized Multi-Protocol Label Switching (GMPLS) protocol suite is emerging as the control plane standard for next generation data networks [1]. Specific signaling protocol objects have been designed to support Label Switched Path (LSP) setup in Wavelength Division Multiplexed (WDM) optical networks, where labels correspond to physical wavelengths [2]. However open issues are still present due to the lack of label preference schemes. Ideally, preference would be assigned to labels which ensure a wavelength continuous path, or reduce either the physical impairments or the collision with concurrent requests. Previous approaches include [3], where wavelength conversions were reduced by only allowing them in the LSP setup attempts following a setup failure. However, this solution increases the signaling message exchanges and the setup time. In [4], pools of flagged wavelengths were introduced for reducing collisions. In [5], a wavelength ranking approach was proposed for multi-domain LSP setup, requiring an additional signaling message and the storage of a perdestination wavelength preference state in each node.

We propose an approach avoiding additional signaling messages and exploiting only non-persistent state information used during LSP setup. Broadening the scope and the results in [6], we show how standard (i.e., the Suggested Label) and novel (i.e., the Suggested Vector) signaling protocol objects can be used to enforce label preference schemes. Many performance targets can be chosen, e.g., Wavelength Converter (WC) usage reduction, physical impairment mitigation, traffic engineering,

Manuscript received July 5, 2006. The associate editor coordinating the review of this letter and approving it for publication was Dr. Nikos Nikolaou. This work was partially supported by the E-Photon/ONe Project.

N. Andriolli, L. Valcarenghi, and P. Castoldi are with Scuola Superiore Sant'Anna, Pisa, Italy (e-mail: \{nick, valcarenghi, castoldi $\} @$ sssup.it).

J. Buron and S. Ruepp are with COM·DTU, Technical University of Denmark, Kgs. Lyngby, Denmark (e-mail: \{jbu, sr\}@com.dtu.dk).

F. Cugini is with CNIT, Pisa, Italy (email: filippo.cugini@cnit.it).

Digital Object Identifier 10.1109/LCOMM.2006.061034. each leading to a different label preference assignment. Here we present a case-study aimed at WC usage reduction.

\section{Label Preference ObJects}

After finding a route using OSPF-TE, a GMPLS signaling session is initiated with an RSVP-TE Path message containing a Generalized Label Request object, issued by each node to the downstream neighbor to notify the label binding request [1]. It may include optional objects, such as the Label Set [2] and the ones described in Sec. II-A and II-B. These objects are stored in the traversed nodes during the LSP setup phase only, and can be discarded afterwards. When the Path message reaches the destination node, a Resv message is sent back carrying a Generalized Label object, where every node indicates to the upstream neighbor the label assigned to the incoming request. When the Resv message reaches the source node, the LSP is established.

\section{A. Suggested Label}

The Suggested Label is a scalar object issued from an upstream node to the downstream neighbor indicating the expected label and was designed to speed up the optical node configuration [2]. We broaden the application scope of the Suggested Label, using it to notify the most preferable label for various targets, such as WC usage reduction, LSP setup request collision avoidance, physical impairment mitigation.

To show how nodes compute Suggested Label values we refer to WC usage reduction case-study. The source node is oblivious of the optimal label to minimize WC, so it simply chooses an available one (with a tie-breaking policy, if more than one can be chosen). Each intermediate node continues suggesting the same label if it is available on the next hop. If not, a label not requiring a $\mathrm{WC}$ in the node is chosen. If a WC is needed in any case, an available label is chosen.

However, the limited information carried in the Suggested Label may lead to suboptimal decisions.

\section{B. Suggested Vector}

To overcome Suggested Label limitations, the Suggested Vector is proposed. It is carried in the Path message and aimed at specifying a preference level for each label in the Label Set. The Suggested Vector scope is general, allowing to indicate any preference metric for ranking the labels. In the case-study aimed at WC usage minimization, the Suggested 


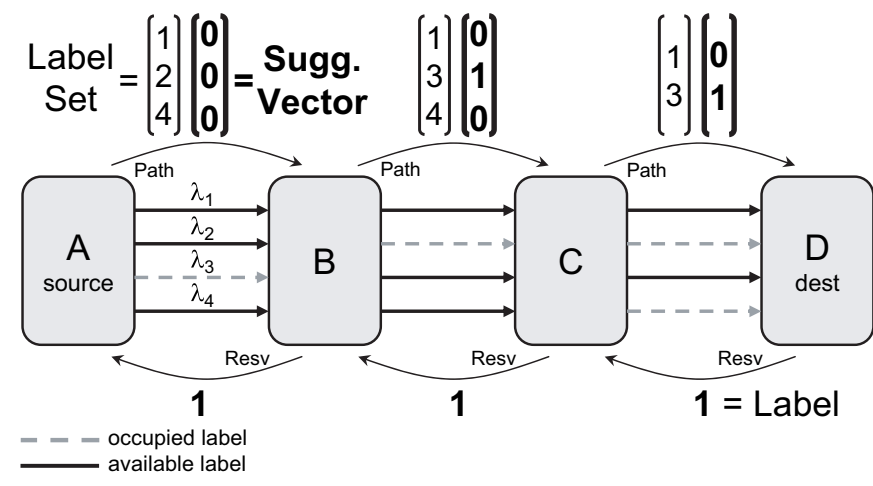

Fig. 1. Example of utilization of the Suggested Vector.

Vector contains the number of WCs needed to use each label in the Label Set on the next hop.

Fig. 1 exemplifies the concept of the Suggested Vector. Source node A fills the Suggested Vector with zeros, because the source node can reach all available labels without WCs. Node B keeps the values for $\lambda_{1}$ and $\lambda_{4}$ unchanged because they are free on its previous hop, but assigns 1 to $\lambda_{3}$, since it is busy on its previous hop. Thus the number of required WCs is computed as one plus the minimum value of the Suggested Vector on the previous hop. Node C keeps the values for $\lambda_{1}$ and $\lambda_{3}$ unchanged. Thanks to the information carried in the Suggested Vector, the destination node D is fully aware of the number of WCs needed for each label in the received Label Set, and can choose $\lambda_{1}$, which minimizes this value.

\section{LABEL PREFERENCE SCHEMES}

Exploiting the previously described protocol objects, two label preference schemes, called SL and SV, have been devised and compared with standard schemes, termed NP and LS.

No Preference scheme (NP): it is the simplest one, not using any protocol object to specify label preferences. When an LSP request reaches the destination, a label free on the last hop is reserved and propagated upstream. If a node cannot propagate the received label because it is busy on the previous hop and a WC is available, a free label on the previous hop is chosen; otherwise the request is blocked.

Label Set scheme (LS): it only uses the Label Set object. At the destination, a label within the received Label Set is reserved. If a node cannot propagate the received label and a WC is available, a free label is chosen within the node's received Label Set; otherwise the request is blocked.

Suggested Label scheme (SL): it uses the Suggested Label together with the Label Set. At the destination, the Suggested Label is reserved. If a node cannot propagate the received label and a WC is available, the previous hop Suggested Label is attempted before the other labels within the Label Set; otherwise the request is blocked.

Suggested Vector scheme (SV): it uses the Suggested Vector together with the Label Set. At the destination, a label with minimum Suggested Vector value is reserved. If a node cannot propagate the received label and a WC is available, a free label with minimum value of the previous hop Suggested Vector is chosen; otherwise the request is blocked.

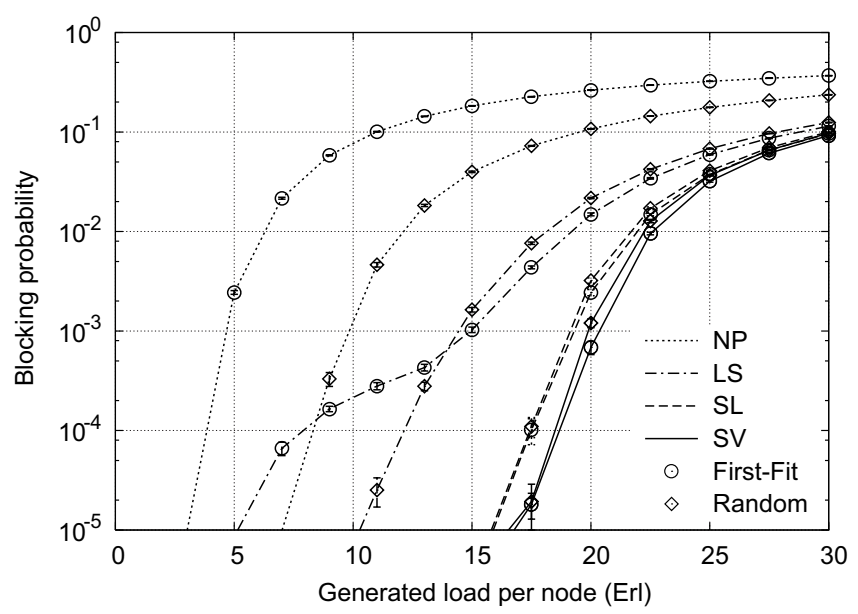

Fig. 2. Blocking probability with $W=32$ and $C=16$.

Each scheme is evaluated with First-fit and Random tiebreaking policies [7]. The performance metrics are the blocking probability, computed as the ratio between the number of blocked requests and the total amount of generated requests, and the WC usage, defined as the average number of active WCs in the network.

\section{Results}

OPNET Modeler [8] has been used for simulations. The studied network is the 15-node 24-link Pan-European topology shown in [9]. $W$ denotes the number of wavelengths per link, while $C$ is the number of WCs per node. LSP requests are generated at each node according to a Poisson process and LSP holding time is exponentially distributed. Shortest path routing is performed considering the links with at least one available wavelength. Blocked requests are rejected without further setup attempts. Plots include confidence intervals at 95\% confidence level, computed from 20 repetitions (each generating at least 125000 requests) for each traffic value.

Fig. 2 and 3 have been obtained with $W=32$ and $C=16$. In Fig. 2, NP blocking probability suddenly rises to a very high value, because the blind wavelength assignment quickly exhausts all WCs. With NP, the First-fit policy performs worse than the Random policy, because the first-fit label chosen at the destination might be already used along the path with higher probability than a randomly chosen one. LS, SL, and SV schemes, exploiting the Label Set object, perform much better than NP.

LS with First-fit presents an early blocking probability rise due to resource contention with concurrent requests, occurring during Resv message processing and at lower loads for Firstfit than for Random. However, for higher loads (when WCs are exhausted) the blocking during Path message processing (due to the absence of labels ensuring a wavelength continuous path) becomes dominant. In this situation, the First-fit policy performs better (in accordance with [7]) because it tends to pack connections on low-indexed wavelengths, leaving more room available on high-indexed wavelengths.

Even though LS blocking probability is much lower than NP, this scheme is significantly outperformed by both SL and 


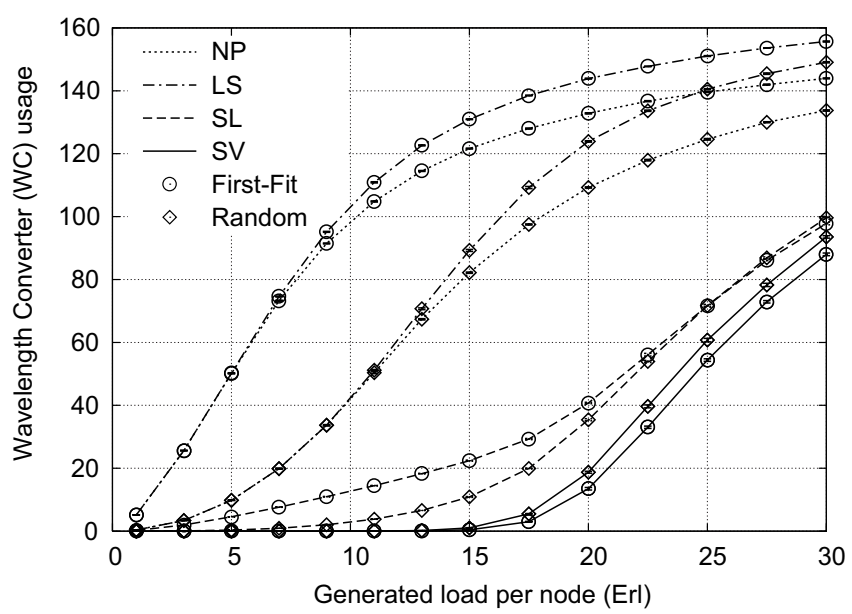

Fig. 3. Wavelength converter (WC) usage with $W=32$ and $C=16$.

SV schemes, due to a more careful WC utilization. With Firstfit tie-breaking policy, SL and SV show an increase in accepted traffic of $24 \%$ and $35 \%$ respectively, at $10^{-3}$ blocking probability. In particular, SV with First-fit policy proves to be the best label preference scheme from the blocking probability standpoint.

Fig. 3 shows that both NP and LS schemes tend to exploit many WCs even for low loads, especially with First-fit tiebreaking policy. For high loads a saturation phenomenon appears, due to the limited amount of WCs per node. At a load of 15 Erl, NP requires 122 and 82 WCs with First-fit and Random policies respectively, while LS requires 131 and 89 WCs with the same policies. With LS, the price to pay for lower blocking is then higher WC usage.

On the contrary, both SL and SV schemes use a significantly smaller amount of WCs. The SL scheme performs better when the Random policy is exploited (only $11 \mathrm{WCs}$ at $15 \mathrm{Erl}$ ). With the First-fit policy instead, the WC utilization grows even for low traffic ( 22 WCs at 15 Erl), because nodes tend to suggest low-indexed wavelengths, which are more likely to be occupied on some of the links along the path. These issues are solved with SV: up to a load of 15 Erl this scheme does not exploit WCs at all. Even for higher loads, SV uses the least amount of WCs among all label preference schemes. Moreover, SV is the only scheme where First-fit reduces WC utilization compared to Random, because in SV ties are broken only among labels minimizing WCs. Under this assumption, the First-fit behavior of packing connections on few wavelength layers does not cause unnecessary WCs, instead it allows a better exploitation of the wavelength domain. Then SL and SV represent very good performance trade-offs, giving both lower blocking probability and lower WC usage.

Fig. 4 shows the blocking probability as a function of $C$ at a load of 15 Erl. Without WCs $(C=0)$, all schemes exploiting the Label Set (LS, SL, and SV) perform the same, as expected. The much worse performance of NP confirms the importance of the Label Set in networks without WCs. However the Label Set alone does not take advantage of the presence of the WCs: LS blocking probability only very slowly decreases for increasing $C$. On the contrary, both SL and $\mathrm{SV}$ show a much quicker blocking decrease. SL reaches the

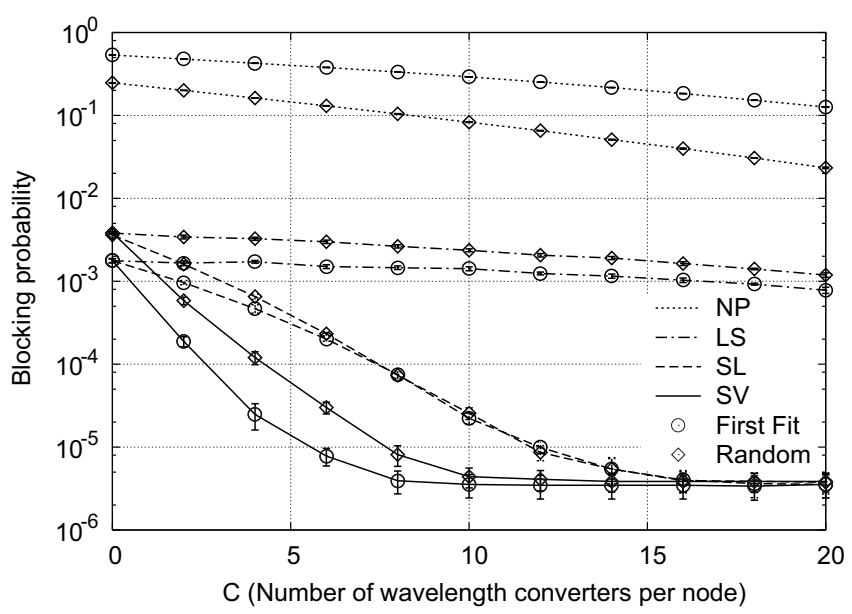

Fig. 4. Blocking probability with $W=32$ and $L o a d=15$ Erl per node.

blocking probability minimum (to which all schemes tend for $C \rightarrow \infty)$ at $C=16$. SV with First-fit has the steepest blocking reduction and requires just $C=10$ to reach the minimum.

\section{CONCLUSion}

In this paper we have proposed enhanced label preference schemes to improve the performance of GMPLS controlled networks. They are enforced during the signaling session exploiting a standard object, the Suggested Label, and a novel object, the Suggested Vector.

In the case-study aimed at WC usage reduction, both SL and SV schemes significantly decrease the blocking probability and the WC usage compared to currently exploited NP and LS schemes. SV with First-fit policy outperforms all the other schemes, minimizing both WC utilization and blocking probability, while SL achieves very good performance without requiring any protocol modification.

\section{REFERENCES}

[1] E. Mannie (ed.), "Generalized Multi-Protocol Label Switching (GMPLS) architecture," RFC 3945, Oct. 2004.

[2] L. Berger (ed.), "Generalized Multi-Protocol Label Switching (GMPLS) signaling functional description," RFC 3471, Jan. 2003.

[3] I. Widjaja and A. Elwalid, "Study of GMPLS lightpath setup over lambdarouter networks," in Proc. ICC'02, pp. 2707-2711.

[4] T. Ozugur, M.-A. Park, and J. P. Jue, "Label prioritization in GMPLScentric all-optical networks," in Proc. ICC'03, pp. 1283-1287.

[5] T. Tachibana and H. Harai, "Aggressive rank accounting for end-toend lightpath establishment in multi-domain WDM networks," in Proc. ICC'06.

[6] N. Andriolli, J. Buron, S. Ruepp, F. Cugini, L. Valcarenghi, and P. Castoldi, "Signaling protocol extensions for converter-saving wavelength assignment in GMPLS optical networks," in Proc. HPSR'06, pp. 349355 .

[7] H. Zang, J. Jue, and B. Mukherjee, "A review of routing and wavelength assignment approaches for wavelength-routed optical WDM networks," Optical Networks Mag., vol. 1, no. 1, pp. 47-60, Jan. 2000.

[8] OPNET Technologies, Inc. (http://www.opnet.com).

[9] A. Giorgetti, N. Andriolli, L. Valcarenghi, and P. Castoldi, "Failure-aware idle protection capacity reuse," in Proc. GLOBECOM'05, pp. 1895-1899. 